\title{
Pregnancy outcome in pre-gestational and gestational diabetic women: a prospective observational study
}

\author{
Mamta Mahajan*, Amit Gupta, Anju Vij, Aanchal Gupta Sharma
}

Dr. Rajendra Prasad Government Medical College, Tanda, Kangra, Himachal Pradesh, India

Received: 25 May 2021

Revised: 22 June 2021

Accepted: 23 June 2021

\section{*Correspondence:}

Dr. Mamta Mahajan,

E-mail: drmamtamahajan@gmail.com

Copyright: ( ) the author(s), publisher and licensee Medip Academy. This is an open-access article distributed under the terms of the Creative Commons Attribution Non-Commercial License, which permits unrestricted non-commercial use, distribution, and reproduction in any medium, provided the original work is properly cited.

\section{ABSTRACT}

Background: India is considered the world capital of diabetes, proper care and management of the same is the demand of society. The present study is carried out to identify the disease burden of GDM/ overt DM among antenatal cases. The main objective was to study the maternal and fetal outcome of diabetes complicating pregnancy.

Methods: The present study was conducted at Dr. Rajendra Prasad Government Medical College at Tanda, District KangraHP from October 2015 to September 2016. All antenatal cases were screened for diabetes by OGTT 75 gm, 2 hour blood glucose level as per DIPSI guidelines and labelled as GDM/ overt DM. Those who fulfilled selection criteria were enrolled in the study. A total of 6452 cases who attended antenatal clinic during the study duration and were screened were selected for the study. 116 cases were found to have GDM / overt DM. Seventy-nine had GDM with OGTT $>140 \mathrm{mg} / \mathrm{dl}$ (DIPSI guidelines) and 37 had overt DM with 2 hours PP >200 mg/dl (WHO criteria). Total 100 cases comprised of study group were followed till delivery to study maternal and fetal outcome.

Results: The prevalence of diabetes in pregnancy was found to be low 1.79\%. GDM was found to be more prevalent than overt diabetes in pregnant women (66\% versus 34\%). Among the antenatal maternal complications observed missed abortion $(11.8 \%$ versus 1.55 ; $\mathrm{p}=0.026)$, polyhydraminos $(26.4 \%$ versus $10.6 \%$; $\mathrm{p}=0.04)$ and preterm labour $(17.6 \%$ versus $4.5 \% ; \mathrm{p}=0.003)$ were significantly more common in overt diabetics than GDM cases. IUFD (8.8\% versus $0 ; \mathrm{p}=0.014$ ) was also significantly more common in overt diabetics than GDM case. RDS was found significantly higher in neonates of overt diabetics as compared to GDM mothers (14.7\% versus $1.5 \%$; $=0.009)$.

Conclusions: Early detection and good glycemia control by MNT and insulin, regular antenatal check-ups, patient counselling and compliance, intrapartum fetal monitoring and early neonatal care are keys to improved outcome.

Keywords: Gestational, Diabetes, Hyperglycemia, Pre-gestational women, Gestational diabetic women

\section{INTRODUCTION}

A global estimate for hyperglycemia in pregnancy (HIP) have brought out that $16.9 \%$ of total pregnancies globally were affected by some form of hyperglycemia in $2013 .{ }^{1}$ This translates to 21.4 million live births at risk of being exposed to a hyperglycemia intrauterine milieu. Southeast Asia region had the maximum prevalence of HIPof $25 \%$ and India is standing tall at $27.5 \%$ against the global average of $16.9 \%$. Country specific estimates have further brought out that India had the highest number of women affected by the HIP with an estimated 5.7 million cases in 2013. Diabetes in pregnancy can be classified into two major types: Gestational diabetes (GDM) -women who have carbohydrate intolerance of variable severity with onset or first recognition during pregnancy., ${ }^{1,2}$ Pregestational or Overt DM (PGDM) - women who were known to have diabetes before pregnancy.

The risk of adverse pregnancy outcome increases continuously with increasing maternal glucose even below the traditional cut-offs for gestational diabetes, with no 
threshold effect found. The findings of the Hyperglycaemia and adverse pregnancy outcomes (HAPO study) have shown continuous relationship between maternal glucose level and adverse outcomes. ${ }^{3}$

Pregnancy aggravates the diabetic state of the mother due to the diabetogenic effect of pregnancy hormones. However, the impact is more severe in the overt diabetic group. Pregnancy in diabetic women leads to disturbed glucose homeostasis, increased insulin requirement, and increased risk of nephropathy and retinopathy in mother. There are several factors that are associated with a greater risk of developing GDM/ overt DM during pregnancy. Women with age $>25$ years, high BMI or obesity, family history of DM, or belonging to ethnic groups like Asian, Pacific Islander, Native American, Hispanic, or black racial have a higher prevalence of DM. Women with past history of GDM or with a previous history of macrosomic baby/fetal loss/ congenital anomalies/ polyhydramnios/ still-birth or persistent glycosuria are found to be at increased risk. ${ }^{4}$ They demand more careful assessment, timely screening, early recognition, intense monitoring, and appropriate management. GDM accounts for $90 \%$ of all cases of diabetes in pregnancy, and if unrecognized and untreated, threatens the lives of both mother and baby. Women with GDM often give birth to macrosomic or large for gestational age (LGA) infants. Pregnancies complicated by GDM also have a 4-fold increased risk of perinatal mortality. Almost 3 million babies are stillborn every year, and GDM is a major contributor to this unacceptable loss of life..$^{5}$ Although GDM is a temporary phenomenon for pregnant women, more than $50 \%$ of women with GDM develop type 2 diabetes within 5-10 years of delivery. ${ }^{6}$ Moreover, infants of women with GDM have a higher prevalence of overweight or obesity as young children and adolescents, and a higher risk of developing type 2 DM later in life. Studies have revealed that women with GDM usually have better maternal and neonatal outcome as compared to overt diabetes group and most of the women with GDM revert back to euglycemic state in postpartum period. ${ }^{7,8}$ Thus, diabetes complicating pregnancy act as a two-way sword- diabetes affecting pregnancy state of the mother and pregnancy affecting the diabetic state of the mother. Since India is considered the world capital of diabetes, proper care, and management of the same is the demand of society. The present study is carried out to identify the disease burden of GDM/ overt DM among antenatal cases in a tertiary care centre in the hilly terrain of North India. The main objective was to study the maternal and fetal outcome of diabetes complicating pregnancy with an idea to fill the knowledge gap.

\section{METHODS}

The present study was a prospective observational study, carried out in the Department of Obstetrics and Gynaecology and Department of Paediatrics, at Dr. Rajendra Prasad Government Medical College at Tanda,
District Kangra, Himachal Pradesh. This was a one-year study conducted from October 2015 to September 2016.

\section{Sample size}

All the pregnant women attending the antenatal clinic or admitted in the antenatal ward and labor room with: Oral glucose tolerance test (OGTT) value $>140 \mathrm{mg} / \mathrm{dl}$. Known case of DM or $2 \mathrm{hr}$ postprandial (PP) value $>200 \mathrm{mg} / \mathrm{dl}$ were eligible to be enrolled for study, after taking written informed consent.

\section{Inclusion criteria}

All the pregnant women attending the antenatal clinic with singleton pregnancy, pregnancy with GDM and pregnancy with overt DM were included in the study.

\section{Exclusion criteria}

Women with multi-fetal gestation, antepartum eclampsia, jaundice complicating pregnancy, placenta praevia, chronic hypertension, known case of heart disease, seizure disorder on antiepileptics, tuberculosis/ bronchial asthma were excluded from the study group.

\section{Methodology}

After initial registration of all the antenatal cases at antenatal clinic, the details of obstetrics/menstrual and medical history were noted and complete general and obstetrics examination was done. This was followed by routine antenatal tests and screening for diabetes by Oral glucose tolerance test (OGTT) 75 gm glucose load irrespective of their last meal timing and blood glucose testing at 2 hours as per Diabetes in Pregnancy Study Group (DIPSI) guidelines at their first, visit. Women with normal values were again given glucose challenge in a similar manner between 24-28 weeks of gestation. Those women attending the antenatal clinic in our hospital for the first time in a later trimester were also screened with the help of DIPSI guidelines. The plasma glucose was estimated by the glucose oxidase-peroxidase (GOD-POD) method. Women with value $\geq 140 \mathrm{mg} / \mathrm{dl}$ and $<200 \mathrm{mg} / \mathrm{dl}$ were labelled as GDM and those with $2 \mathrm{hr} \mathrm{PP} \geq 200 \mathrm{mg} / \mathrm{dl}$ were labelled as overt diabetes (WHO criteria)

All GDM and overt DM cases who were ready to participate in the study and fulfilling selection criteria were enrolled in the study. The participants were admitted to the antenatal ward and were treated with diet and/or insulin therapy. GDM women were advised medical nutrition therapy (MNT) for 2 weeks in consultation with the dietician of the hospital. Those who achieved desired target levels while maintaining fasting plasma glucose (FPG) $\leq 90 \mathrm{mg} / \mathrm{dl}$ and peak post-meal glucose $\leq 120 \mathrm{mg} / \mathrm{dl}$ were discharged with weekly follow-up. Those who did not respond by maintaining FPG $\leq 90 \mathrm{mg} / \mathrm{dl}$ and $2 \mathrm{hr}$ PP $\leq 120 \mathrm{mg} / \mathrm{dl}$ were advised insulin in consultation with the 
medical department of the hospital. All of them were followed until delivery Figure 1.

Maternal complications like missed or threatened abortion, polyhydramnios, pregnancy induced hypertension (PIH), pre-eclampsia $(\mathrm{PE})$, preterm labor, premature rupture of membrane (PROM) and any other morbidity was recorded in predesigned proforma. The period of gestation at the time of delivery and mode of delivery including instrumental delivery or cesarean section were recorded.

A detailed examination of the new born was done. The APGAR score at 1 minute and 5 minutes of birth and the resuscitation required were noted. Fetal parameters such as birth weight, any features of macrosomia, congenital malformation, blood sugar levels for incidence of hypoglycemia (at 0,1,2,3 hour of life followed by 6 hourly blood glucose monitoring till 48 hours of life: as per standard protocol followed in the pediatrics department of college), respiratory distress syndrome, hypocalcemia, polycythemia, neonatal jaundice, early neonatal death etc. were recorded in consultation with Paediatrician. The management and investigations of all the new-borns were done till their discharge and were recorded on predesigned proforma.

\section{Statistical analysis}

The complete data was collected in a Microsoft excel sheet and statistical analysis of data was done with the help of Epi info software. Mean values and percentages were collected to analyze the study.

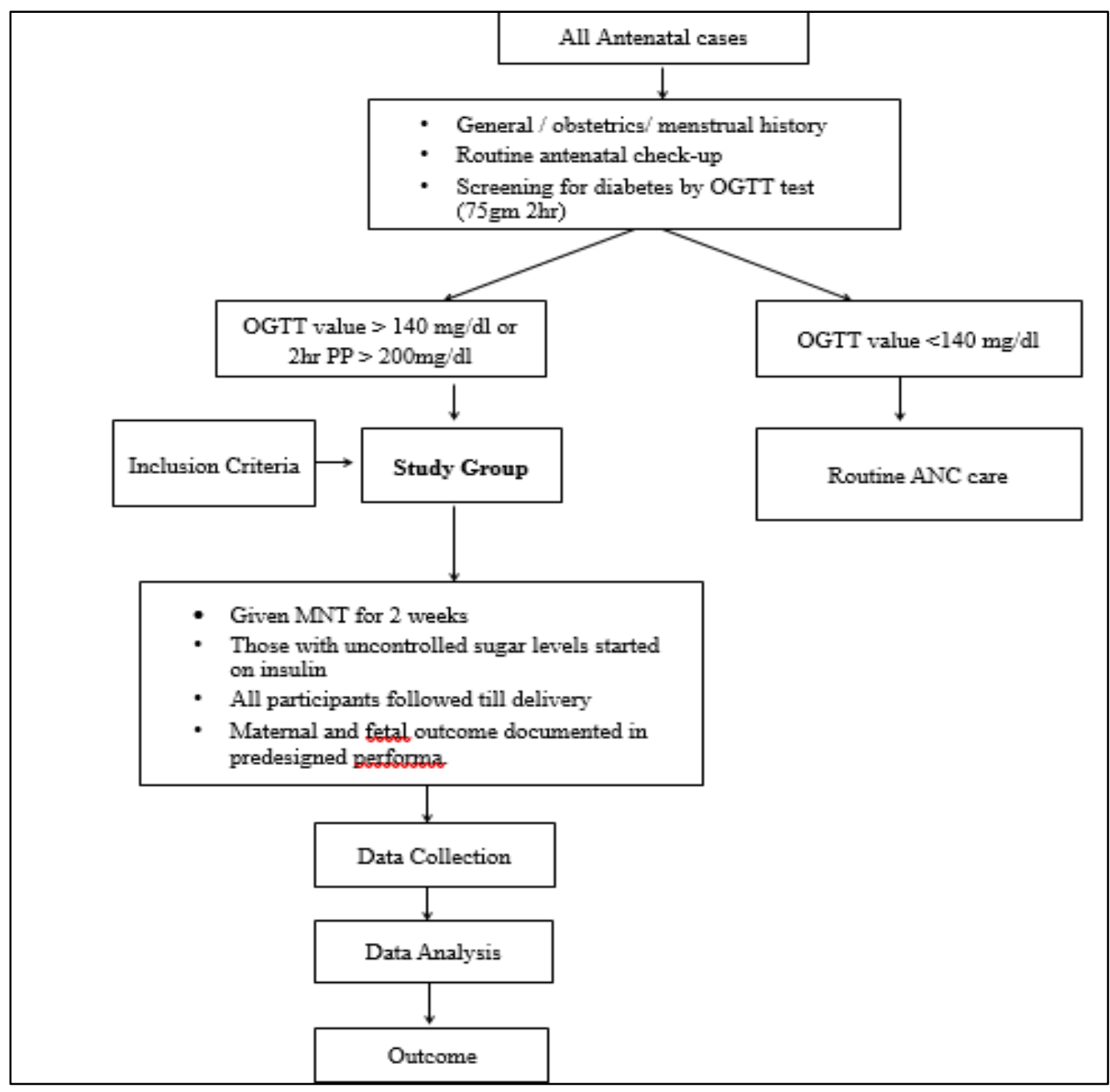

Figure 1: Methodology flow chart.

\section{Ethical issues}

There was no drug trial or human /animal experiment involved. All the women were treated in their best interest in consultation with a consultant on duty. All the deliveries and cesarean sections were attended by a paediatrician. The Investigators and supervisors were well aware of the guidelines for ethics in the biomedical research document 
of ICMR (2006), Helsinki Declaration (modified 2000), and the policy of the Institutional Ethics Committee of Dr. RPGMC Tanda.

\section{RESULTS}

The present study was conducted to study the maternal and fetal outcome of diabetes in pregnancy in Department of Obstetrics and Gynaecology and Department of Paediatrics, at Dr Rajendra Prasad Government Medical College at Tanda, District Kangra, Himachal Pradesh from
October 2015 to September 2016 after the due approval of the 'Institutional Ethics Committee'.

A total of 6452 antenatal cases who were screened as per DIPSI guidelines were selected. 116 cases were found to have GDM / overt DM. Thus, prevalence of diabetes complicating pregnancy was found to be $1.79 \%$. Out of 116, 79 were labelled as GDM (OGTT >140 mg/dl) and 37 had overt DM ( $2 \mathrm{hr}$ PP $>200 \mathrm{mg} / \mathrm{dl}$, WHO criteria). 2 cases did not consent for participation in study and 14 cases were lost to follow up. Total 100 cases comprised of study group.

Table 1: Characteristics of singleton women with gestational and pregestational diabetes mellitus as well age and neonatal birth weight at delivery.

\begin{tabular}{|c|c|c|c|}
\hline Characteristic & $\begin{array}{l}\text { Gestational diabetes } \\
(n=66)\end{array}$ & $\begin{array}{l}\text { Pregestational } \\
\text { diabetes }(n=34)\end{array}$ & $\begin{array}{l}\text { Statistical } \\
\text { comparison } \\
\text { between groups }\end{array}$ \\
\hline Mean Maternal Age (years) & 29.5 & 31.4 & $\mathrm{p}=0.010$ \\
\hline No. (\%) Primigravidae & $25(37.9)$ & $8(23.5)$ & $\mathrm{p}=0.148$ \\
\hline $\begin{array}{l}\text { Mean Pre pregnancy BMI * } \\
(\mathrm{Kg} / \mathrm{m} 2) \text { No. }(\%)\end{array}$ & $24.72 \pm 3.75$ & $25.27 \pm 5.18$ & $\mathrm{p}=0.588$ \\
\hline Lean (18.5-22.9) & $20(30.3)$ & $10(29.4)$ & NA \\
\hline Overweight (23.0-27.5) & $14(21.2)$ & $6(17.6)$ & NA \\
\hline Obese $(>27.5)$ & $32(48.5)$ & $15(44.2)$ & NA \\
\hline No. (\%) Previous still birth & $10(15.1)$ & $9(26.5)$ & $\mathrm{p}=0.07$ \\
\hline Mean HbA1C level & $5.81 \pm 0.305 \%$ & $8.188 \pm 1.71 \%$ & $\mathrm{p}=0.001$ \\
\hline $\begin{array}{l}\text { Mean gestational age at current delivery } \\
\text { (Weeks) No. }(\%)\end{array}$ & $37.4 \pm 2.3$ & $34.2 \pm 3$ & $\mathrm{p}=0.006$ \\
\hline \multicolumn{4}{|l|}{ Gestational age at delivery } \\
\hline$<34$ weeks & $1(1.5)$ & $2(5.8)$ & \\
\hline $34-36+6$ & $9(13.6)$ & $11(32.3)$ & \\
\hline $37-42$ & $4(81.8)$ & $1(44.1)$ & \\
\hline $\begin{array}{l}\text { Mean birth weight at current delivery } \\
\text { (gms.) }\end{array}$ & $3205 \pm 073$ & $2803 \pm 098$ & $\mathrm{p}=0.019$ \\
\hline
\end{tabular}

* WHO classification for Asian population

Table 2: Distribution of cases according to antenatal maternal complications.

\begin{tabular}{|c|c|c|c|c|c|c|}
\hline \multirow{2}{*}{ Type of complication } & \multirow{2}{*}{ Total } & \multicolumn{2}{|c|}{ GDM (66) } & \multicolumn{2}{|c|}{ Overt DM (34) } & \multirow{2}{*}{$P$ value } \\
\hline & & $\mathbf{N}$ & $\%$ & $\mathbf{n}$ & $\%$ & \\
\hline Missed abortion & 5 & 1 & 1.5 & 4 & 11.8 & $0.026^{\#}$ \\
\hline UTI & 7 & 3 & 4.5 & 4 & 11.8 & 0.180 \\
\hline Candidiasis & 9 & 6 & 9 & 3 & 8.8 & 0.245 \\
\hline DKA & 1 & 0 & 0 & 1 & 2.9 & 0.160 \\
\hline D. retinopathy & 2 & 0 & 0 & 2 & 5.8 & 0.216 \\
\hline D. nephropathy & 1 & 0 & 0 & 1 & 2.9 & 0.734 \\
\hline Preterm labour & 9 & 3 & 4.5 & 6 & 17.6 & $0.030^{\#}$ \\
\hline PPROM & 8 & 3 & 4.5 & 5 & 14.7 & 0.076 \\
\hline Polyhydraminos & 16 & 7 & 10.5 & 9 & 26.4 & $0.040^{\#}$ \\
\hline Pre-eclampsia & 12 & 6 & 9 & 6 & 17.6 & 0.212 \\
\hline PROM & 9 & 7 & 10.5 & 2 & 5.8 & 0.434 \\
\hline Total & 79 & 37 & & 42 & & \\
\hline Uneventful & $37 / 100$ & 30 & 45 & 7 & 20.5 & $0.004^{\#}$ \\
\hline
\end{tabular}

\# p $<0.05$ statistically significant 
Table 3: Distribution of cases according to antenatal fetal complications.

\begin{tabular}{|c|c|c|c|c|c|c|}
\hline \multirow{2}{*}{ Type of complication } & \multirow{2}{*}{ Total } & \multicolumn{2}{|c|}{ GDM (66) } & \multicolumn{2}{|c|}{ Overt DM (34) } & \multirow{2}{*}{$P$ value } \\
\hline & & $\mathbf{N}$ & $\%$ & $\mathbf{n}$ & $\%$ & \\
\hline Congenital malformation & 4 & 1 & 1.5 & 3 & 8.8 & 0.067 \\
\hline IUFD & 3 & 0 & 0 & 3 & 8.8 & $0.014^{\#}$ \\
\hline Macrosomia & 15 & 11 & 16.7 & 4 & 11.8 & 0.884 \\
\hline IUGR & 2 & 0 & 0 & 2 & 5.8 & 0.216 \\
\hline Uteroplacental insufficiency & 2 & 0 & 0 & 2 & 5.8 & 0.216 \\
\hline Total & 26 & 12 & 14 & & & \\
\hline Uneventful & $76 / 100$ & 53 & 80.3 & 23 & 67.6 & $0.034^{\#}$ \\
\hline
\end{tabular}

\# $\mathrm{p}<0.05$ statistically significant

Table 4: Mode of delivery and intrapartum complications.

\begin{tabular}{|llllllll|}
\hline Type of complication & $\begin{array}{l}\text { Total } \\
(\mathbf{1 0 0})\end{array}$ & $\mathbf{n}$ & $\mathbf{9}$ & $\begin{array}{l}\text { Gvert DM (34) } \\
\text { Overt DM } \\
\text { II }\end{array}$ & $\begin{array}{l}\text { Overt DM } \\
\text { I }\end{array}$ & \% & P value \\
\hline NVD & 44 & 33 & 50 & 10 & 1 & 32.3 & 0.12 \\
\hline LSCS & 34 & 21 & 31.8 & 11 & 2 & 38.2 & 0.521 \\
\hline Instrumental Delivery & 14 & 10 & 15.2 & 4 & 0 & 11.7 & 0.644 \\
\hline Intrapartum Hypoglycemia & 1 & 0 & 0 & 0 & 1 & 2.9 & 0.738 \\
\hline Acute Fetal Distress & 9 & 6 & 9 & 3 & 0 & 8.8 & 0.965 \\
\hline Meconium Stained Liqour & 9 & 6 & 9 & 3 & 0 & 8.8 & 0.965 \\
\hline Stillbirth & 2 & 2 & 3 & 0 & 0 & 0 & 0.305 \\
\hline Shoulder Dystocia & 2 & 2 & 3 & 0 & 0 & 0 & 0.305 \\
\hline
\end{tabular}

Table 5: Neonatal complications.

\begin{tabular}{|c|c|c|c|c|c|c|c|}
\hline \multirow[b]{2}{*}{ Neonatal complication } & \multirow[b]{2}{*}{$\begin{array}{l}\text { Total } \\
(92)\end{array}$} & \multicolumn{2}{|c|}{ GDM } & \multicolumn{3}{|c|}{ OVERT DM } & \multirow[b]{2}{*}{ P value } \\
\hline & & $\mathbf{N}$ & $\%$ & $\begin{array}{l}\text { OVERT } \\
\text { DM II }\end{array}$ & $\begin{array}{l}\text { OVERT } \\
\text { DM I }\end{array}$ & $\%$ & \\
\hline RDS & 6 & 1 & 1.5 & 5 & 0 & 14.7 & $0.009^{\#}$ \\
\hline Hypoglycemia & 13 & 8 & 12 & 5 & 0 & 14.7 & 0.716 \\
\hline NNJ & 15 & 9 & 13.6 & 5 & 1 & 17.6 & 0.595 \\
\hline LGA & 8 & 4 & 6.06 & 3 & 1 & 11.7 & 0.602 \\
\hline Macrosomia (>4000gm) & 15 & 12 & 18.2 & 3 & 0 & 8.8 & 0.2 \\
\hline LBW & 10 & 4 & 6.06 & 5 & 1 & 17.6 & 0.067 \\
\hline Neonatal Period Uneventful & 44 & 36 & 54.5 & 8 & & 23.5 & $0.003^{\#}$ \\
\hline
\end{tabular}

\# $\mathrm{p}<0.05$ statistically significant.

Of 100 cases, 66 cases had GDM and 34 cases had overt DM. Of 34 overt DM cases, 31 had Type II DM and 3 had Type I DM (IDDM) Figure 2. Characteristics of women in both the groups are shown in Table 1 .

In present study around $88 \%$ cases were with age $>25$ years. The mean age was found to be higher in overt diabetics as compared to GDM women, 31.4 years versus 27.5 years respectively with $\mathrm{p}=0.010$ (Table 1$)$.

Women with GDM had lower BMI compared to overt diabetics, $24.72 \pm 3.75 \mathrm{~kg} / \mathrm{m} 2$ and $27 \pm 5.18 \mathrm{~kg} / \mathrm{m} 2$ respectively with p value 0.588 (Table 1 ).

In present study 31 cases $(31 \%)$ had positive family history of diabetes. Positive family history in mother was significantly more commonly associated with GDM (21,
$31.8 \%)$ than overt DM group $(4,11.7 \%)$ with $\mathrm{p}$ value 0.028 .

Past history of infertility, early pregnancy failures and term IUFD were enquired, the difference was statistically significant only in relation with history of term IUFD, significantly higher in overt diabetics with $\mathrm{p}$ value 0.03 (Table 1).

HbA1c was found higher in overt diabetics compared to women with GDM, 8.18 $\pm 1.7 \%$ and $5.89 \pm 0.305 \%$ respectively with $\mathrm{p}=0.001$ (Table 1 ).

Total 47 cases, 45 in GDM (68.2\%) and 2 in overt DM group $(5.9 \%)$ were managed with MNT alone while insulin therapy was supplemented in 41 cases, 16 in GDM group $(24.2 \%)$ and 25 in overt DM group (73.5\%) to achieve desired glycemic control.(Fig3)12 cases did not receive any treatment and constituted those cases who 
were unbooked unsupervised cases and came in labour (2) or had some adverse pregnancy outcome like missed abortion (5)/ CMF (4)/ IUFD (1), where pregnancy needed to be terminated before instituting any treatment.

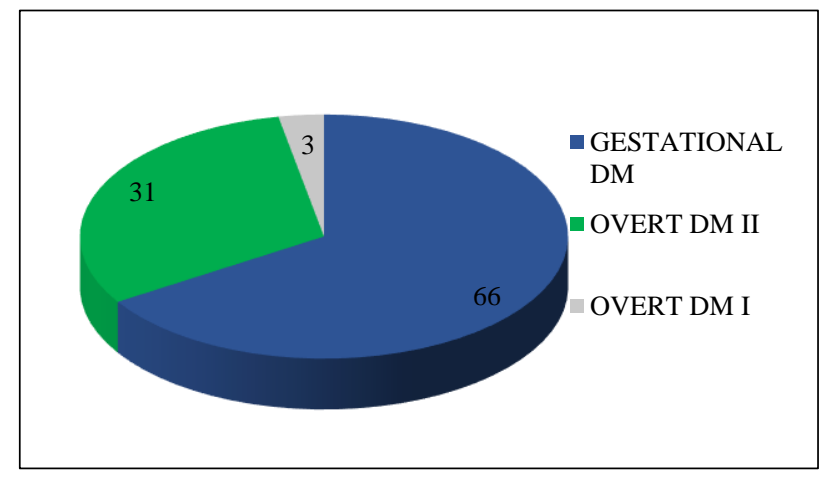

Figure 2: Distribution of cases according to type of diabetes.

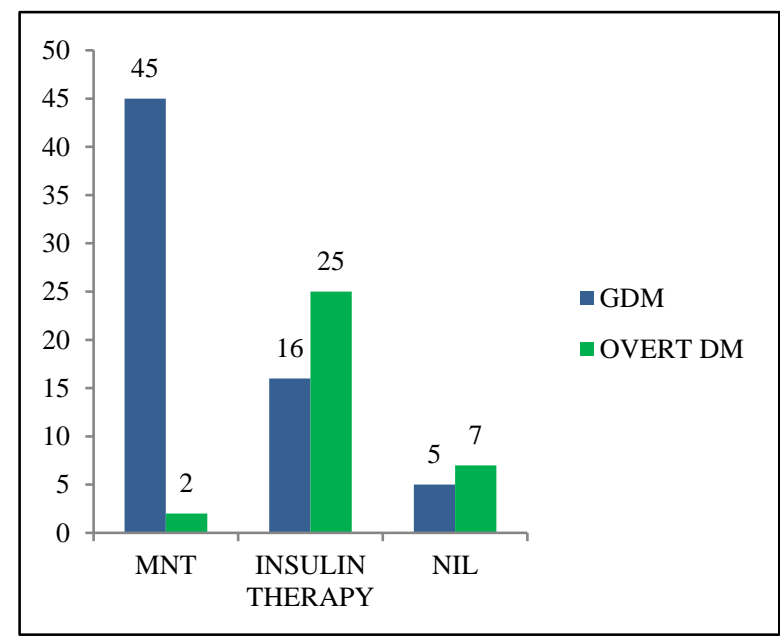

Figure 3: Distribution of cases according to treatment instituted.

Antenatal maternal complications developed in 63 cases (63\%) (Table 2). Antenatal maternal complications noted were missed abortion $(5,5 \%)$, UTI $(7,7 \%)$, candidiasis $(9$, 9\%), Diabetic Ketoacidosis (1, 1\%), Diabetic Retinopathy $(2,2 \%)$, Diabetic Nephropathy $(1,1 \%)$, preterm labour ( 9 , $9 \%)$, preterm PROM $(8,8 \%)$, polyhydraminos $(16,16 \%)$, pre-eclampsia $(12,12 \%)$ and PROM $(9,9 \%)$. Antenatal period was uneventful in significantly higher number of GDM cases $(30,45 \%)$ compared to overt diabetics $(7$, $20.5 \%$ ) with $\mathrm{p}=0.004$.

Among fetal complications observed were CMF in 4 cases, IUFD in 3, macrosomia in 15 cases, IUGR with Uteroplacental insufficiency in 2 cases. (Table 3 ).

CMF (4) in fetus were observed in 1case of GDM (1.5\%) and 3 cases $(8.8 \%)$ of overt diabetics. IUFD (3) was observed in 3 cases of overt diabetics $(8.8 \%)$. Macrosomia (15) was observed in 11 cases of GDM $(16.7 \%)$ and 4 cases of overt DM (11.8\%). IUGR with Utero-placental insufficiency (2) were observed in 2 cases of overt diabetics $(5.8 \%)$.

Of total cases, 8 had 1st and 2nd trimester losses, 3 had early preterm delivery (POG $<34$ weeks), 20 had late preterm delivery (POG 34 weeks - 36+6weeks) and 69 had term delivery (POG 37 weeks - 42 weeks). The mean gestational age at termination of pregnancy was significantly earlier in overt DM group as compared to

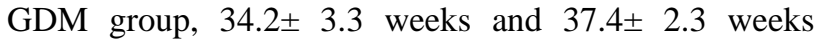
respectively with $\mathrm{p}$ value 0.006 (Table 1 ).

34 cases were delivered by LSCS, 14 cases had instrumental delivery while 44 cases had normal vaginal delivery. Suction and evacuation were done in 5 cases for missed abortion. LSCS rate were higher in women with overt diabetes $(13,38.2 \%)$ as compared to GDM (21, $31.8 \%$ ) with $\mathrm{p}$ value 0.521 . Instrumental delivery was found higher in GDM than overt diabetics 15.2 versus $11.8 \%, \mathrm{p}=0.644$ (Table 4)

Among intrapartum fetal complications observed, 9 fetuses developed acute fetal distress (9\%), 9 had meconium-stained liquor (9\%), 2 were stillborn $(2 \%)$ and 2 had shoulder dystocia (2\%) (Table 4).

Shoulder dystocia (2) were observed in 2 cases of GDM $(3 \%)$ and none in overt diabetics with $p$ value 0.305 . Still birth (2) was observed in 2 cases of GDM group (3\%) and none in overt diabetics $(0 \%)$ with $\mathrm{p}=0.305$.

In present study out of total 92 new-borns, 10 had birth weight $<2500 \mathrm{gm}(10 \%), 67$ had birth weight between 2500 to $4000 \mathrm{gm}(67 \%)$ and 15 had birth weight $>4000 \mathrm{gm}$

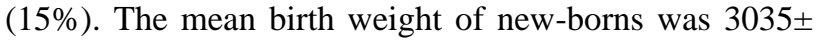
$855 \mathrm{gm}$. New-borns born to GDM mothers were found significantly heavier than overt diabetics, with mean birth weight $3250 \pm 730 \mathrm{gm}$ and $2820 \pm 980 \mathrm{gm}$ respectively with $\mathrm{p}=0.019$. (Table 1).

Macrosomia (birth weight $>4000 \mathrm{gm}$ ) (15) was observed in 12 neonates of GDM mothers $(18.2 \%)$ and 3 neonates of overt diabetes $(8.8 \%)$ with p value 0.214 . LGA (birth weight greater than 90th centile) (8) was noted in 4 newborns of GDM mothers $(6.06 \%)$ and 4 new-borns of overt diabetics $(11.7 \%)$ with $\mathrm{p}=0.602$. LBW (birth weight $<2500$ gm) (10) was detected in 4new-borns of born to GDM mothers $(6.06 \%)$ and 6 new-borns born to mothers with overt diabetes $(17.6 \%)$ with p value 0.067 (Table 5).

RDS (6) was noted in significantly higher in neonates of overt diabetics $(5,14.7 \%)$ compared to GDM women (1, $1.5 \%$ ) with $\mathrm{p}=0.009$. Neonatal hypoglycemia (13) was observed in 8 new-borns of GDM mothers $(12 \%)$ and 5 new-borns of overt diabetics $(14.7 \%)$ with $\mathrm{p}=0.716$. NNJ (15) was observed in 9 new-borns of GDM mothers $(13.6 \%)$ and 6 new-borns of overt diabetics (17.6\%) with $\mathrm{p}$ value 0.595 (Table 5). 
Out of 92 cases, one had multiple congenital anomalies incompatible with life, 3 had intrauterine fetal demise and 2 were still born. Rest 86 neonates were normal and euglycemic at the time of discharge from the hospital.

\section{DISCUSSION}

The present study prevalence of diabetes complicating pregnancy was found to be $1.79 \%$ (116/6452). Kavyashree et al 2014 in Mysore, Karnataka reported similar prevalence rate of $1.64 \%(50 / 3044)$ of which $90 \%$ had GDM, $6 \%$ were overt diabetics type I and $4 \%$ were overt diabetics type II. $^{9}$ In studies conducted in various other parts of country, prevalence of GDM was found to be $2.6 \%$ by Gajjar et al 2005 in Baroda, Gujarat; 3.6\% by Sawant et al 2012 in Loni, Maharashtra. ${ }^{10,11}$

The mean age was found to be higher in overt diabetics as compared to GDM women, 31.4 years versus 27.5 years respectively with statistically significant difference with $\mathrm{p}=0.010$. Age $>25$ years is a recognised risk factor for developing GDM in pregnancy. In present study around $88 \%$ cases were with age $>25$ years. In a study by Shefali et al 2006 mean age in overt diabetics was $29 \pm 6$ years and in GDM was $29 \pm 5$ years which was comparable to our study. ${ }^{12}$ In present study 31 cases were found to have positive family history of diabetes (31\%). 23 cases of GDM $(34.8 \%)$ and 8 cases of overt diabetes $(23.4 \%)$ had associated positive family history of DM. It was close to that found by study by Patil et al 2014 and, Kalra et al 2013. ${ }^{13,14}$

In the present study, LSCS rate were higher in women with overt diabetes $(13,38.2 \%)$ as compared to GDM (21, $31.8 \%$ ) with p value 0.521 . In studies by Wahabi et al 2014 and Abu Heijaet al 2015 caesarean rate in overt diabetics and GDM was $46.6 \%$ versus $26 \%$ and $60.3 \%$ versus $27.9 \%$ respectively. ${ }^{15,16}$ While in a study by Patil et al 2014 caesarean rate was reported higher in GDM than overt diabetics (48\% versus $30 \%) .{ }^{17}$ Also, in several studies conducted in various parts of country on GDM, very higher rate of caesarean section were reported than our study and the rate has increased dramatically in recent past.

In present study instrumental delivery rate were found higher in GDM than overt diabetics $15.2 \%$ versus $11.8 \%$, $\mathrm{p}=0.644$. In studies by Gajjaret al 2005, Kalyani et al20 2014 and Swaroop et al 2015 rate of instrumental delivery was $11 \%, 12 \%$ and $18 \%$ in GDM respectively which was comparable to our study. ${ }^{10,19}$ In a study by Kalraet al 2013 reported rate of instrumental delivery was $3 \%$ which was lower than our study. ${ }^{14}$

In present study pre-eclampsia was observed in 12 cases, 6 in GDM group (9\%) and 6 in overt DM group (17.6\%). Thus, pre-eclampsia was found higher in mothers with overt DM with $\mathrm{p}=0.212$. Studies by Sugiyama et al 2014 and Abu Heijaet al 2015 reported rate of pre-eclampsia of $6.1 \%$ versus $10.1 \%$ and $7.8 \%$ versus $17.2 \%$ in GDM and overt diabetics respectively which was comparable to our study. ${ }^{16,19}$

In present study polyhydraminos was observed in 16 cases, 7 in GDM (10.6\%) and 9 in overt diabetics (26.4\%). This was significantly higher in overt diabetics with $\mathrm{p}$ value 0.040. In studies by Patil et al 2014 and Abu Heijaet al 2015 incidence was $5.2 \%$ versus $7.6 \%$ and $6.8 \%$ versus $4.9 \%$ in overt diabetics and GDM respectively. ${ }^{16,17}$ Sawant et al 2012 and Kavyashreeet al 2014 reported incidence of polyhydraminos in GDM $16.6 \%$ which was higher than our study. ${ }^{9,11}$ Poor glycemic control and poor compliance to treatment especially in third trimester increases the rate of polyhydraminos. In present study preterm labour (PTL) was observed in 9 cases, 3 in GDM (4.5\%) and 6 in overt diabetic cases $(17.6 \%)$ significantly higher in overt diabetics with $\mathrm{p}$ value 0.03 . This finding was comparable with other studies done by Wahabi et al 2014 (18\% versus $8.5 \%)$ and Heijaet al 2015 (25.9\% versus $9.5 \%) .^{15,16}$

In present study $\mathrm{CMF}$ in fetus was observed in 4 cases (4\%), 1 in GDM (1.5\%) and in 3 fetuses of overt diabetic mothers $(8.8 \%)$ with $\mathrm{p}=0.067$. While Sawant et al 2012 and Swaroop et al 2015 reported CMF rate of $11.11 \%$ and $13.6 \%$ respectively in GDM which was higher than our study. ${ }^{11,17} \mathrm{CMF}$ in DM is associated with severity of hyperglycemia during period of organogenesis. So, CMF are found higher in overt diabetics. Pinter et al studied the mechanism by which hyperglycemia produced dysmorphogenesis and proposed it to be hyperglycemia induced yolk sac failure and resulting compromise of nutrient transfer and oxygenation to the early embryo. ${ }^{18}$ Good glycemic control in preconception and early pregnancy has major role in prevention of $\mathrm{CMF}$ in pregnancies complicated by diabetes.

In present study new-borns born to GDM mothers were heavier than overt diabetics, the difference was found to be statistically significant with $\mathrm{p}=0.019$. In present study macrosomia (birth weight $>4000 \mathrm{gm}$ ) was detected in 15 neonates (92). 12 neonates having macrosomia were born to GDM mothers (18.2\%) and 3 neonates with macrosomia were born to mothers with overt diabetes $(8.8 \%)$. Kalra et al 2013 reported comparable rate of $18 \%$ in their study in GDM. ${ }^{14}$ In present study 8 new-borns were LGA (birth weight greater than 90th centile) $(8 \%)$. This was noted in 4 new-borns of GDM mothers $(6.06 \%)$ and 4 new-borns of overt diabetics $(11.7 \%)$ with $\mathrm{p}=0.602$. It is very important to find large for gestational age neonates as they remain at a higher risk of hypoglycemia in neonatal period and also at a higher risk of developing childhood obesity and early onset diabetes mellitus later in life. In a study Shefali et al 2006 reported the incidence of $27.6 \%$ and $19.2 \%$ in GDM and overt diabetics respectively which was higher than our study. ${ }^{12}$

In present study RDS was observed significantly higher in neonates of overt diabetics with $\mathrm{p}=0.009$. Heija et al 2015 also reported higher rate of RDS in overt diabetics $(8.5$ versus $2.6 \%$ ) while Patil et al 2014 reported higher rate in 
GDM compared to overt diabetics (11.6 versus 5.1\%). ${ }^{16,17}$ In similar study by Kavyashree et al 2014 incidence of RDS was $12.5 \%$ which was comparable to our study. ${ }^{9}$

\section{Limitations}

The present study was an institutional based study. Thus the prevalence of diabetes in pregnancy found in this study may not be exact representation of the study population. There was no control group taken in the study thus comparison with non-diabetic data lacking in the present study.

\section{CONCLUSION}

Timely screening, early detection of GDM and overt DM in pregnancy, regular close follow ups and institutional delivery of these pregnant women will improve pregnancy outcome. Preconceptional counselling of women with risk of hyperglycaemia in pregnancy ensures that she enters pregnancy with an optimal state of health. The need of the hour is to make preconceptional counselling a 'done thing' in India too as in western countries. Large multicentric population based study should be carried out to know the disease burden in the study population and to further strengthen the health system to combat the adverse effect of diabetes on maternal and fetal outcome.

\section{Funding: No funding sources}

Conflict of interest: None declared

Ethical approval: The study was approved by the Institutional Ethics Committee

\section{REFERENCES}

1. Gauriguata L, Linnenkamp U, Beagley J. Global estimates of the prevalence of hyperglycemia in pregnancy for 2013 for IDF Diabetes Atlas. Diabetes Res Clin Pract. 2011.

2. American College of Obstetricians and Gynecologists: Gestational diabetes mellitus. Practice Bulletin. 2013;137.

3. Metzger BE, Lowe LP, Dyer AR. Hyperglycemia and adverse pregnancy outcomes. $\mathrm{N}$ Eng $\mathrm{J}$ Med. 2008;358:1991-2002.

4. American Diabetes Association: Standards of medical care in diabetes - 2013. Diabetes Care. 2013;1:S1166.

5. Cousens S, Stanton C, Blencowe H. National, Regional, and Worldwide Estimates of Stillbirth Rates in 2009 with Trends Since 1995: A Systematic Analysis. Lancet. 2011.

6. Kim C, Newton KM, Knopp RH. Gestational Diabetes and the Incidence of Type 2Diabetes: A Systematic Review. Diabetes Care. 2002;25.

7. Ray JG, Vermeulen MJ, Shapiro JL, Kenshole AB. Maternal and neonatal outcomes in pregestational and gestational diabetes mellitus, and the influence on maternal obesity and weight gain: the DEPOSIT study. Diabetes Endocrine Pregnancy Outcome Study in Toronto. QJM. 2001;94(7):347-56.

8. Kitzmiller JL, Dang-Kilduff L, Taslimi MM. Gestational diabetes after delivery. Short-term management and long-term risks. Diabetes Care. 2007;30(2):S225-35.

9. Kavyashree KS, Suma KB, Prashanth SN. A Clinical Study of Maternal Complications and Perinatal Outcomes in Diabetes Complicating Pregnancy. Sch. J. App Med. Sci. 2014;2(6A):1954-7.

10. GajjarF, Maitra NK. Intrapartum and perinatal outcomes in women with gestational diabetes and mild gestational hyperglycemia. J Obstet Gynecol.2005;55(2):135-7.

11. Sawant AP, Naik SS, Nagarkar VD. Glycemic status during pregnancy in Gestational Diabetic \& NonGestational Diabetic women \& its effect on maternal $\&$ fetal outcome. Journal of Dental \& Allied Sciences. 2012;1(2):57-62.

12. Shefali AK, Kavitha M, Deepa R, Mohan V. Pregnancy outcomes in pre-gestational and gestational diabetic women in comparison to nondiabetic women--A prospective study in Asian Indian mothers (CURES-35). J Assoc Physicians India. 2006;54:613-8.

13. Patil SK, Pandey PD, Kshirsagar NS. Comparison of Pregnancy Outcomes in Indian Women Complicated withPregestational and Gestational Diabetes Mellitus. International Journal of Recent Trends in Science and Technology. 2014;9(3):457-60.

14. Kalra P, Kachhwaha CP, Singh HV. Prevalence of gestational diabetes mellitus and its outcome in western Rajasthan. Indian $\mathbf{J}$ Endocrinol Metab. 2013;17(4):677-80.

15. Wahabi HA, Fayed A, Esmaeil SA. Maternal and Perinatal Outcomes of Pregnancies Complicated with Pregestational and Gestational Diabetes Mellitus in Saudi Arabia. J Diabetes Metab. 2014;5(7):399-403.

16. Abu Heija AT, Al- Bash M, Mathew M. Gestational and Pregestational Diabetes Mellitus in Omani Women Comparison of obstetric and perinatal outcomes. Sultan Qaboos University Med J. 2015;15(4):496-500.

17. Swaroop N, Rawat R, Lal P, Pal N, Kumari K, Sharma P. Gestational diabetes mellitus: study of prevalence using criteria of diabetes in pregnancy study group in India and its impact on maternal and fetal outcome in a rural tertiary institute. Int $\mathrm{J}$ Reprod Contracept ObstetGynecol. 2015;4(6):1950-3.

18. Pinter E, Reece EA, Leranth CZ. Yolk sac failure in embryopathy due to hyperglycaemia. Teratology. 1986;33(1):73-84.

19. Sugiyama T, Saito M, Nishigori H, Nagase S, Yaegashi N, Sagawa N et al. Japan Diabetes and Pregnancy Study Group. Comparison of pregnancy outcomes between women with gestational diabetes and overt diabetes first diagnosed in pregnancy: a retrospective multi-institutional study in Japan. Diabetes Res Clin Pract. 2014;103(1):20-5. 
20. Kalyani KR, Jajoo S, Hariharan C, Samal S. Prevalence of gestational diabetes mellitus, its associated risk factors and pregnancy outcomes at a rural setup in Central India. Int J Reprod Contracept Obstet Gynecol. 2014;3(1):219-24.
Cite this article as: Mahajan M, Gupta A, Vij A, Sharma AG. Pregnancy outcome in pre-gestational and gestational diabetic women: a prospective observational study. Int J Reprod Contracept Obstet Gynecol 2021;10:3040-8. 